\title{
Comparisons in heart rate readings between the Bioconnected wireless exercise earpiece and a Polar T31-Coded Chest Strap during a GXT
}

TREVOR R. HIGGINS ${ }^{1,2} \longleftarrow$, THOMAS LLOYD ${ }^{1}$, KENNETH MANALANSAN ${ }^{1}$, SARA MCDONALD ${ }^{1}$, BEN THOMAS 1

${ }^{1}$ Australian College of Physical Education, Australia

${ }^{2}$ Sports Performance and Research Training Academy, Australia

\begin{abstract}
Purpose. The advent of mobile technology has provided a convenient method of measuring heart rate in clinical settings and athletic training with limited support. Therefore the purpose of this research was to evaluate the validity of an earpiece HR monitoring device against a previously validated chest strap HR monitoring device. Method. A convenient sample of college students $(n=15)$, performing a modified Bruce protocol, provided 25 data samples. Heart rates obtained from both the Bioconnected wireless exercise earpieces and a Polar T31-Coded Chest Strap where compared by correlation and coefficient. The Bioconnected wireless exercise earpieces were considered to be valid if the correlation between the recorded heart rate of the Bioconnected device and the corresponding heart rate Polar T31-Coded Chest Strap measurement was $r \geq 0.90$. Results. Five samples were corrupted due to displacement of chest $\operatorname{strap}(n=3)$ and dislodgement of earpiece $(n=2)$ during testing, as such data from those tests were excluded from the correlation analysis. The remaining 20 data samples provided mean totals of $521 \pm 117 \mathrm{HR}$ data points (earpiece) and $517 \pm 118 \mathrm{HR}$ data points (chest strap). A strong correlation ( $r=0.97)$ between the Bioconnected wireless exercise earpieces and a Polar T31-Coded Chest Strap. Conclusions. The results of this study show that the HR measurements of the Bioconnected wireless exercise earpieces and the Polar Chest Strap are highly correlated, supporting the Bioconnected wireless exercise earpieces in monitoring HR during a GXT on a treadmill in healthy adults. However, as exercise transitioned from walking to jogging, at times, both devices had problems with displacement and loss of HR signal, suggesting the need for improved
\end{abstract}

\footnotetext{
Corresponding author. Australian College of Physical Education, Australia.

E-mail: thiggins@acpe.edu.au

Submitted for publication January 2017

Accepted for publication October 2017

Published in press May 2018

JOURNAL OF HUMAN SPORT \& EXERCISE ISSN 1988-5202

(c) Faculty of Education. University of Alicante

doi:10.14198/jhse.2018.133.05
} 
methods of securing both devices. Future research of the Bioconnected wireless exercise earpiece is required to evaluate performance in varying environments, levels of low light and increased background noise. Key words: HEART RATE MONITORING, VALIDITY, POLAR MONITOR, PHOTOPLETHYSMOGRAPHY, PPG.

\section{Cite this article as:}

Higgins, T., Lloyd, T., Manalansan, K., McDonald, S., \& Thomas, B. (2018). Comparisons in heart rate readings between the Bioconnected wireless exercise earpiece and a Polar T31-Coded Chest Strap during a GXT. Journal of Human Sport and Exercise, 13(3), 530-540. doi:https://doi.org/10.14198//hse.2018.133.05 


\section{INTRODUCTION}

A vital physiological measurement, Heart Rate $(\mathrm{HR})$ can be employed to measure exercise intensity or prescribe exercise, either based on a percentage of an individual's HRmax or HR reserve (Crouter, Albright, \& Bassett Jr, 2004). Furthermore, as HR is linearly related to oxygen uptake for dynamic activities it can provide a reasonable estimate of energy expenditure (EE) during exercise (Crouter, et al., 2004; Spierer, Rosen, Litman, \& Fujii, 2015). HR was traditionally measured via the radial pulse manually for a 30 or 60 second time interval (Cheatham, Kolber, \& Ernst, 2015). However, with the advent of commercial wrist and belt monitors, professional athletes and the general population are able to more readily monitor heart rate intensity whilst training (Crouter, et al., 2004; Engström, Ottosson, Wohlfart, Grundström, \& Wisén, 2012; Terbizan, Dolezal, \& Albano, 2002). It has been highlighted that approximately 25 million wearable HR monitoring devices were sold in 2015 alone, with wrist-based devices accounting for a large proportion of those sales (Wallen, Gomersall, Keating, Wisløff, \& Coombes, 2016). Spierer et al. (2015) suggested the great popularity of wrist-based devices is due to their convenience and their non-invasive approach. However, Wallen et al. (2016) cautions the accuracy of these devices, indicating the need for evaluation of the validity of the increasing number of HR monitors entering the market (Goodie, Larkin, \& Schauss, 2000).

Of the literature available from sport science databases, evaluating the validity of portable HR monitors, the majority evaluate monitors from the leading manufacturer, Polar Electro, Inc. Finland (Baig, Gholamhosseini, \& Connolly, 2013; Crouter, et al., 2004). Polar HR monitors have been reported to be valid in monitoring HR when compared with electrocardiograms, the gold standard of monitoring HR (Baig, et al., 2013; Crouter, et al., 2004). Further support indicating the validity of Polar monitors has been provided with reports of up to 99\% accuracy rate at maximal HR (Coolbaugh, Anderson, Wilson, Hawkins, \& Amsterdam, 2014), and a variance of $0.05 \%$ between ECG and Polar devices (Vanderlei, Silva, Pastre, Azevedo, \& Godoy, 2008). Therefore it can be assumed that Polar HR monitors are valid in the monitoring and analysis of HR.

One major limitation of chest strap devices identified in the literature, is associated with perceived discomfort of chest strap devices (Leboeuf, Aumer, Kraus, Johnson, \& Duscha, 2014). Therefore, recommendations have been provided for the development of devices that alleviate the discomfort associated with chest strap HR monitors (Leboeuf, et al., 2014). These recommendations have coincided with the entry of wrist based devices and earpiece devices onto the market (Wang et al., 2016). Initial reports indicate variable accuracy of wrist based devices, that wrist based devices accuracy is best at rest and diminishes with exercise (Wang, et al., 2016). Limitations of wrist based devices include the variable accuracy reported and the inability of models investigated to conduct continuous HR monitoring (Wang, et al., 2016), therefore chest strap devices should be used when continuous and accurate HR monitoring is required (Wang, et al., 2016).

The absence of bone and predominantly compromising of cartilage and blood suggests the ear is suitable for continuous HR monitoring with photoplethysmography (PPG) (Budidha \& Kyriacou, 2014; Park, Jang, Park, \& Youm, 2015). The length of the ear canal is roughly $2.5 \mathrm{~cm}$, with a diameter of approximately $7 \mathrm{~mm}$ with exceedingly sensitive skin, (Park et al., 2015), with PPG technology, the earpiece sensors detect how much light is reflected from the subcutaneous blood vessels (Budidha \& Kyriacou, 2014). PPG uses optoelectronic features to isolate red and infrared wavelengths and light refraction to dictate blood flow (Schafer \& Vagedes, 2013; Spierer, et al., 2015). Initial research into earpiece HR monitoring devices, indicates they may be an accurate tool for monitoring HR. LeBoeuf et al. (2014) demonstrated an earpiece HR monitor to be both accurate and convenient when compared with ECG during cardiopulmonary exercise testing. HR measurements between ECG and earpiece HR monitor almost completely overlapped each other (Leboeuf, et al., 2014). In addition, Park et al. (2015) identified an earpiece HR monitoring device to be 
significantly accurate across various intensities of exercise. However, several limitations may occur with earpiece devices, including skin irritation, difficulties connecting to additional devices, bulky sensors and hygiene (Chan, Estève, Fourniols, Escriba, \& Campo, 2012). Furthermore, the sensors have been reported to become displaced, that HR measurements can be disrupted via noise and low light levels and for wires to cause distress and pain (Aarts et al., 2013). In relation to the accuracy and convenience of earpiece devices (Leboeuf, et al., 2014; Park, et al., 2015)and limitations identified (Aarts, et al., 2013), Spierer et al. (2015) suggests further research to validate earpiece HR monitoring devices using PPG technology, is required.

Therefore, the aim of this study was to evaluate the validity of a Bioconnected wireless exercise earpiece (BioConnected, Sydney Olympic Park, Australia) monitoring HR via the ear canal, using PPG, against the previously validated Polar FT1 HR monitor and a Polar T31-Coded chest strap (Polar OY, Finland).

\section{METHOD}

\section{Participants}

A convenient sample of college students free from known injury and/or illness, in the previous four weeks and from the Australian College of Physical Education (ACPE), volunteered to participate in this research (Table 2). Participants were asked to abstain from food and caffeine intake for $2 \mathrm{~h}$ immediately preceding the test. They were also asked not to drink alcohol or exercise at a high intensity for the $24 \mathrm{~h}$ period prior to the test. Participants provided informed written consent in-line with approval of the study granted by the ACPE Ethics Committee conforming to the principles of the declaration of Helsinki.

\section{Procedure}

Participants performed a modified Bruce Protocol, providing 25 separate data samples, on a Life Fitness treadmill (LifeFitness, Illinois, USA). All participants were fitted with both the Bioconnected wireless exercise earpieces and a Polar T31-Coded Chest Strap. The Bioconnected wireless exercise earpieces were connected to the Bioconnected iOS smartphone application via Bluetooth. The Polar FT1 HR monitor and a Polar T31-Coded chest strap was synced together to monitor HR. To elicit a HR response, the Bruce Protocol, a maximal graded exercise testing (GXT), was utilized for this research (Eston et al., 2012). As smartphone devices have been reported to be reliable measurements of HR (Silva \& UI-Haq, 2013), each HR monitor was linked to an Apple iPhone via Bluetooth using the Wahoo Fitness Application with HR measurements measured in beats per minute (BPM) continuously across the Bruce protocol. The data collected via the iPhone was extracted via email for analysis with the Bioconnected wireless exercise earpieces the device under test.

The Bruce protocol GXT (see Table 1) was adapted from previous research evaluating HR response to exercise (Eston et al., 2012). Each subject performed the same GXT commencing at a speed of $4 \mathrm{kph}$ at 0 degree incline. An incremental increase of $1 \mathrm{kph}$ every two minutes for 12 minutes or until volitional fatigue was reached.

\section{Statistical analysis}

The heart rates obtained from both the Bioconnected wireless exercise earpieces and a Polar T31-Coded Chest Strap where compared by correlation and coefficient of determination in Microsoft Excel 2010 (Microsoft Corporation, New Mexico, U.S) with the following formula.

$$
\operatorname{Correl}(X, Y)=\frac{\sum(x-\bar{x})(y-\bar{y})}{\sqrt{\sum(x-\bar{x})^{2} \sum(y-\bar{y})^{2}}}
$$


The Bioconnected wireless exercise earpieces were considered to be valid if the correlation between the recorded heart rate of the Bioconnected device and the corresponding heart rate Polar T31-Coded Chest Strap measurement was $r \geq 0.90$ (Terbizan, et al., 2002).

Table 1. Bruce protocol.

\begin{tabular}{cccc}
\hline Level & Time $(\min )$ & Incline $(\mathrm{deg})$ & Speed $(\mathrm{kph})$ \\
\hline 1 & $0: 00-1: 59$ & 0.0 & 4 \\
2 & $2: 00-3: 59$ & 0.0 & 5 \\
3 & $4: 00-5: 59$ & 0.0 & 6 \\
4 & $6: 00-6: 59$ & 0.0 & 7 \\
5 & $7: 00-7: 59$ & 0.0 & 8 \\
6 & $8: 00-8: 59$ & 0.0 & 9 \\
7 & $9: 00-9: 59$ & 0.0 & 10 \\
8 & $10: 00-10: 59$ & 0.0 & 11 \\
9 & $11: 00-12: 00$ & 0.0 & 12 \\
\hline
\end{tabular}

\section{RESULTS}

Table 2. Participant characteristics.

\begin{tabular}{ccccc}
\hline & $\mathrm{N}$ & $\begin{array}{c}\text { Age yr } \\
( \pm \mathrm{SD})\end{array}$ & Height $\mathrm{m}( \pm \mathrm{SD})$ & $\begin{array}{c}\text { Weight kg } \\
( \pm \mathrm{SD})\end{array}$ \\
\hline Male & 12 & $20.6( \pm 1.92)$ & $1.77( \pm 0.06)$ & $75.78( \pm 10.25)$ \\
Female & 3 & $20.3( \pm 0.58)$ & $1.68( \pm 0.01)$ & $68.33( \pm 2.08)$ \\
Group & 15 & $20.6( \pm 1.76)$ & $1.76( \pm 0.07)$ & $75.78( \pm 9.94)$ \\
\hline
\end{tabular}

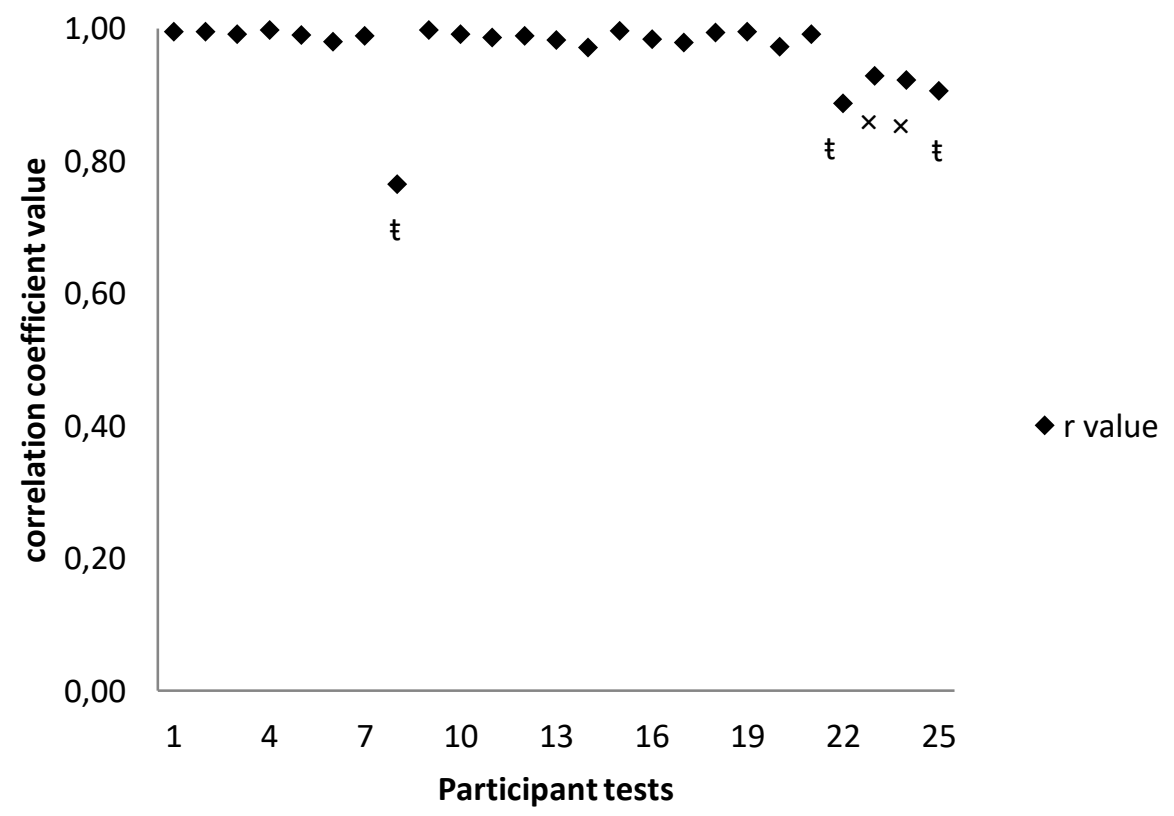

$\ddagger$ indicates displacement of Polar strap occurred at intervals during the Bruce Protocol.

$\mathrm{x}$ indicates dislodgement of ear piece occurred at intervals during the Bruce Protocol.

Figure 1. Average R Value of each participant test; mean scores $0.97 \pm 0.05$. 


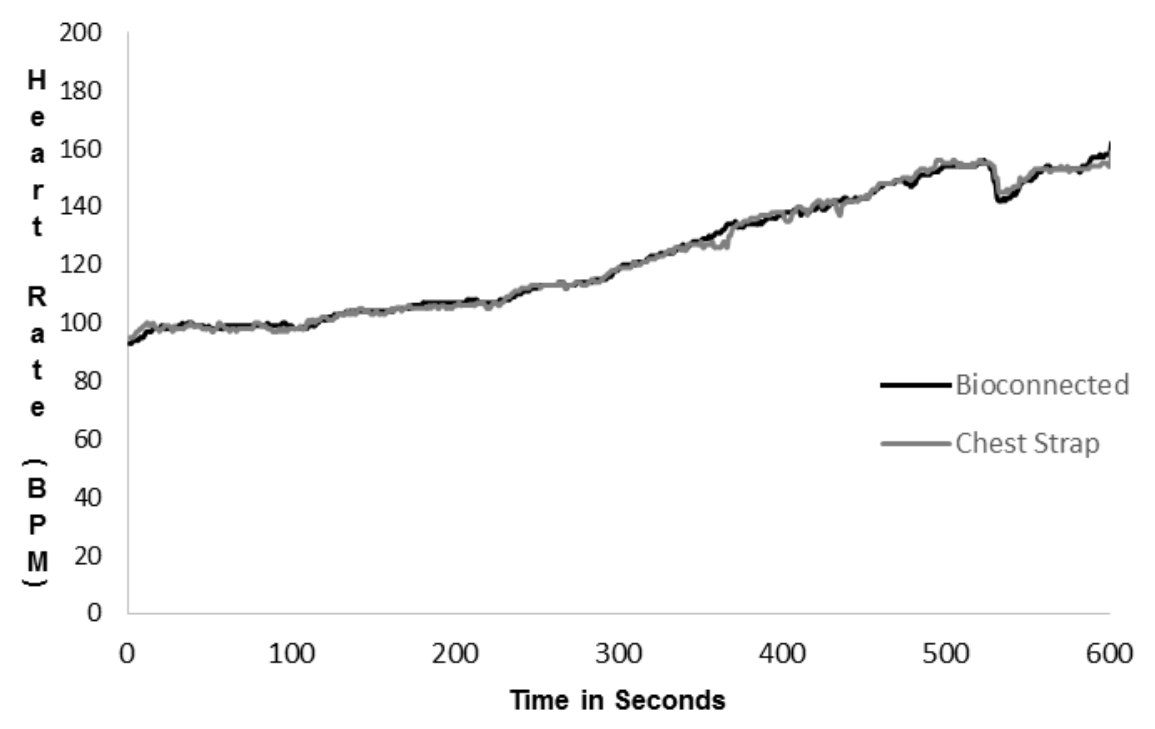

Figure 2. Comparison of mean HR measurements and mean $r$ value between the Bioconnected Wireless Exercise Earpieces and Polar Chest Strap, where no displacement of either strap or earpiece occurred.

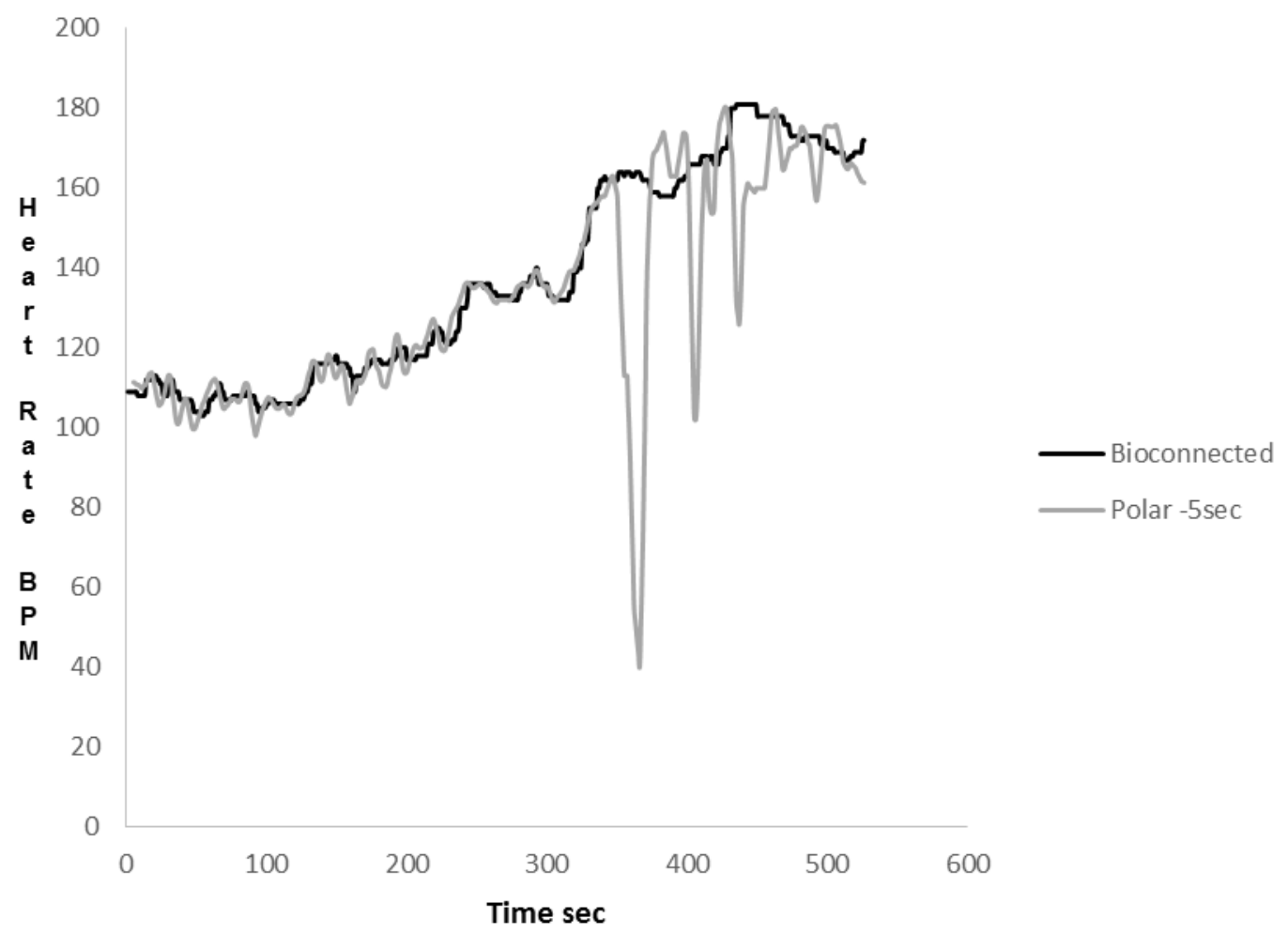

Figure 3. The line graph of trial 8, displaying the Polar chest strap displacement. 


\section{DISCUSSION}

The advent of mobile technology has provided a convenient method of measuring pulse rate in clinical settings and with athletic training without validated support (Cheatham, et al., 2015). Therefore, the purpose of this research was to evaluate the validity of an earpiece HR monitoring device against a previously validated chest strap HR monitoring device. A convenient sample of college students, performing a modified Bruce protocol provided 25 data samples. Of the 25 data samples, five samples were corrupted due to displacement of chest strap $(n=3)$ and dislodgement of earpiece $(n=2)$ during testing, as such data from those tests were excluded from the correlation analysis. The remaining 20 data samples provided a mean total of $521 \pm 117 \mathrm{HR}$ data points (earpiece) and $517 \pm 118 \mathrm{HR}$ data points (chest strap). This study identified a strong correlation between the Bioconnected wireless exercise earpieces and a Polar T31-Coded Chest Strap $(r=0.97)$ supporting the Bioconnected wireless exercise earpieces as a valid HR monitor.

The results in this study support previous evaluations of the validity of earpiece HR monitors (Goodie, et al., 2000; Leboeuf, et al., 2014; Park, et al., 2015). Previous studies exemplified a tight correlation between HR data points measured by an earbud sensor and ECG sensors with HR data points almost completely overlapping (Leboeuf, et al., 2014). In this study, the overlapping of HR readings was also observed between the chest strap and earpiece device. Both devices were closely linked for the first 350 seconds of the Bruce protocol. There was a slight discrepancy when participants transferred from walking to a slow jog, with the Bioconnected wireless exercise earpieces demonstrated a gradual increase of $\mathrm{HR}$, whereas the Polar Chest Strap shows a sudden increase of HR. This may have been a result of the algorithm and averaging across 5 seconds with the chest strap, whereas the Bioconnected earpiece used continuous monitoring. However, this discrepancy was for a relative short period and $<10 \mathrm{bpm}$ as the overall correlation between both devices was reported $r=0.97$, well above the $r \geq 0.90$ previously established for validity of HR monitoring devices (Terbizan, et al., 2002).

The similarity between the Bioconnected wireless exercise earpieces and the Polar Chest Strap aligns with research from (Parak \& Korhonen, 2014). Using such derived algorithms, both HR monitoring devices in this study delivered accurate results. As stated by Parak and Korhonen (2014), these derived algorithms to calculate HR are valid, however are dependent on the intensity of the activity being performed. According to Goodie et al. (2000) and Parak and Korhonen (2014), the very slight discrepancies in HR measurements is likely affiliated with the difference associated with the derived algorithms used by each device which would explain the discrepancy identified at 360 seconds into the Bruce protocol. Additionally, Goodie et al. (2000) stated it is possible that the derived algorithms used by Polar HR monitoring devices use a methodical and minor overestimation of HR measurements. In this study, it is possible that discrepancies that occurred between both devices were a result of this overestimation of HR. However, despite the slight discrepancy, indications are that they are unlikely to be significant in a clinical setting (Goodie, et al., 2000).

It is likely that the data samples in figure 1 showing high correlation $(r=0.98-1.00)$ are correlated from the derived algorithms. On average figure 1 does not display any significant discrepancies in HR measurements between the chest strap and the earpiece. The greatest difference in HR was no greater than $10 \mathrm{bpm}$ and occurred once at approximately 360 seconds, where exercise intensity increased. Therefore, whilst the derived algorithms may differentiate between the two devices, the HR measurements from the two devices are highly correlated.

Despite the close relationship identified between the two devices, limitations were identified during the study. At times difficulties with connection and displacement of either device became a factor in the results. Results 
shown in figure 1 identify data samples that have lower $r$ values and coefficient, in contrast to the other results. Temporary displacement of either device resulted in loss of HR resulting in larger discrepancies between the devices reducing $r$ values. Figure 3 displays the impact on HR values as a result of temporary displacement of the chest strap leading to the temporary loss of signal.

Figure 3 displays the line graph of HR readings during trial 8. In this instance, at the transition from walking to jogging, the participant had issues with the chest strap. The chest strap began to slip from its position, subsequently losing HR signals. Although the participant adjusted the strap whilst continuing the protocol, the movement of the strap continued throughout the remainder of the trial, resulting in the lowest $r=v a l u e$ of the study ( $r=0.58)$. Four other trials experienced similar issues, although not to the same extent. Two of the trials experienced problems with movement of the chest strap and two trials experienced problems with the earpiece dislodging. Although, each experienced problems, the extent and number of times HR readings were lost, the extent was minor in comparison to trail 8. However, although not as severe as trial 8, trials 2225 all reported lower $r$ values indicating that the loss of HR signal does impact correlation values between devices.

On occasion, the Bioconnected earpiece and the chest strap both required readjustment to deliver accurate $\mathrm{HR}$ measurements. Although minor discrepancies in HR measurements during minor displacements would have little significance in a clinical setting, in one data sample (trial 8, figure 1) the displacement of the chest strap showed a large decrease in HR leading to a low coefficient. Such a decrease in HR during moderate intensity exercise is considered extremely abnormal indicating the decrease was a result of chest strap displacement (Ehrman, Gordon, Visich, \& Keteyian, 2013). However, the majority of data samples showed constant overlapping (figure 2) of HR between the Bioconnected wireless exercise earpieces and Polar T31Coded chest strap.

The occasional problems identified in this study in relation to device displacement, lends to further issues previously identified with chest strap devices (Segerståhl \& Oinas-Kukkonen, 2011). Segerstahl and OinasKukkonen (2011) reported that users of chest strap devices found them to be awkward, moving and uncomfortable. As a result, individuals would refuse to use the chest strap whilst exercising (Segerståhl \& Oinas-Kukkonen, 2011). Although in this study, the comfort of an earpiece HR monitor and the chest strap, was not evaluated which resulted in participants rarely commented on the comfort of each device, casual observation by the researchers noted more time was dedicated towards adjusting of the Polar chest strap device as opposed to the Bioconnected wireless exercise earpieces. It should be noted that the Bioconnected wireless exercise earpieces were fitted by the researchers, whereas participants were self-fitting the Polar chest strap. The lack of control with fitting and positioning of the Polar chest strap may be the reasoning for the increased adjustments of the Polar chest strap observed. Irrespective of comfort, the high correlation already discussed indicates the Bioconnected wireless exercise earpiece to be as effective as the Polar Chest Strap in HR monitoring during a GXT on a treadmill.

The results of this study further demonstrate PPG as a technological advancement which delivers accurate HR monitoring, which Schafer and Vagedes (2013) have previously highlighted as a valid form of measuring HR. However, Aarts et al. (2013) believe PPG still requires further development as it is possible that HR monitoring devices using PPG measurements are influenced by low levels of light and noise (Aarts, et al., 2013). In this study, the testing laboratory had sufficient levels of lighting; with moderate levels of background noise. Future research should evaluate varying levels of lighting and noise and the impact on PPG, specifically minimum lighting levels and noise levels for accurate monitoring of $\mathrm{HR}$. 


\section{CONCLUSION}

In conclusion, the Bioconnected wireless exercise earpieces accurately measured HR and its response to exercise in relation to the Polar T31-Coded chest strap. The results of this study show that the HR measurements of the Bioconnected wireless exercise earpieces and the Polar Chest Strap are highly correlated, which supports the use of the Bioconnected wireless exercise earpieces in monitoring HR during a GXT on a treadmill in healthy adults. However, as exercise intensity increased, specifically during the transition from walking to jogging, on occasion, both devices had problems with displacement. In regards to displacement of devices and lost HR signal, alternative methods of securing both the chest strap and the earpiece should be investigated. Furthermore, future research projects should aim for a larger sample size, varying environments and modes of exercise and use a greater variation of HR monitoring devices such as wrist-based devices. In addition, future studies should consider targeting individuals who may exhibit chronic health issues such as heart disease which may have an influence on monitoring the accuracy of HR.

\section{SUPPORTING AGENCIES}

No financial support was received for the purpose of this study.

\section{REFERENCES}

Aarts, L., Jeanne, V., Cleary, J. P., Lieber, C., Stuart Nelson, J., Oetomo, S. B., et al. (2013). Non-contact heart rate monitoring utilizing camera photoplethysmography in the neonatal intensive care unit - $\mathrm{A}$ pilot study. Early Human Development, 89(12), 943-948. https://doi.org/10.1016/i.earlhumdev.2013.09.016

Baig, M., Gholamhosseini, H., \& Connolly, M. (2013). A comprehensive survey of wearable and wireless ECG monitoring systems for older adults. Medical \& Biological Engineering \& Computing, 51(5), 485495. https://doi.org/10.1007/s11517-012-1021-6

Budidha, K., \& Kyriacou, P. (2014). The human ear canal: Investigation of its suitability for monitoring photoplethysmographs and arterial oxygen saturation. Physiological Measurement, 35(2), 111-128. https://doi.org/10.1088/0967-3334/35/2/111

Chan, M., Estève, D., Fourniols, J.-Y., Escriba, C., \& Campo, E. (2012). Smart wearable systems: Current status and future challenges. Artificial Intelligence in Medicine, 56(3), 137-156. https://doi.org/10.1016/i.artmed.2012.09.003

Cheatham, S. W., Kolber, M. J., \& Ernst, M. P. (2015). Concurrent validity of resting pulse-rate measurements: A comparison of 2 smartphone applications, the polar $\mathrm{h} 7$ belt monitor, and a pulse oximeter with bluetooth. Journal of Sport Rehabilitation, 24, 171-178. https://doi.org/10.1123/jsr.2013-0145

Coolbaugh, C., Anderson, I., Wilson, M., Hawkins, D., \& Amsterdam, E. (2014). Evaluation of an Exercise Field Test Using Heart Rate Monitors to Assess Cardiorespiratory Fitness and Heart Rate Recovery in an Asymptomatic Population. PLoS One, 9(5). https://doi.org/10.1371/journal.pone.0097704

Crouter, S. E., Albright, C., \& Bassett Jr, D. R. (2004). Accuracy of polar S410 heart rate monitor to estimate energy cost of exercise. Medicine Science Sports Exercise, 36(8), 1433-1439. https://doi.org/10.1249/01.MSS.0000135794.01507.48

Ehrman, J. K., Gordon, P. M., Visich, P. S., \& Keteyian, S. J. (2013). Clinical Exercise Physiology (3rd ed.). Champaign, IL: Human Kinetics. 
Engström, E., Ottosson, E., Wohlfart, B., Grundström, N., \& Wisén, A. (2012). Comparison of heart rate measured by Polar RS400 and ECG, validity and repeatability. Advances in Physiotherapy, 14, 115122. https://doi.org/10.3109/14038196.2012.694118

Eston, R., Evans, H., Faulkner, J., Lambrick, D., Al-Rahamneh, H., \& Parfitt, G. (2012). A perceptually regulated, graded exercise test predicts peak oxygen uptake during treadmill exercise in active and sedentary participants. European Journal of Applied Physiology, 112(10), 3459-3468. https://doi.org/10.1007/s00421-012-2326-8

Goodie, J., Larkin, K., \& Schauss, S. (2000). Validation of the Polar Heart Rate Monitor for Assessing Heart Rate During Physical and Mental Stress. Journal Of Psychophysiology, 14(3), 159-164. https://doi.org/10.1027//0269-8803.14.3.159

Leboeuf, S. F., Aumer, M. E., Kraus, W. E., Johnson, J. L., \& Duscha, B. (2014). Earbud-based sensor for the assessment of energy expenditure, heart rate, and VO2max. Medicine Science Sports Exercise, 46(5), 1046-1052. https://doi.org/10.1249/MSS.0000000000000183

Parak, J., \& Korhonen, I. (2014). Evaluation of wearable consumer heart rate monitors based on photopletysmography. Paper presented at the 36th Annual International Conference of the IEEE Engineering in Medicine and Biology Society, Chicago, IL, USA. https://doi.org/10.1109/EMBC.2014.6944419

Park, J., Jang, D., Park, J. W., \& Youm, S. (2015). Wearable sensing of in-ear pressure for heart rate monitoring with a piezoelectric sensor. Sensors (Basel), 15(9), 23402-23417. https://doi.org/10.3390/s150923402

Schafer, A., \& Vagedes, J. (2013). How accurate is pulse variability as an estimate of heart rate variability? A review on studies comparing photoplethysmographic technology with an electrocardiogram. International Journal of Cardiology, 166(1), 15-29. https://doi.org/10.1016/i.jicard.2012.03.119

Segerståhl, K., \& Oinas-Kukkonen, H. (2011). Designing personal exercise monitoring employing multiple modes of delivery: Implications from a qualitative study on heart rate monitoring. International Journal Of Medical Informatics, 80(12), e203-e213. https://doi.org/10.1016/.j.jmedinf.2011.08.011

Silva, R., \& UI-Haq, N. (2013). Monitoring heart rate with common market smart-phones for identifying potential signs that may lead to sudden death. Blekinge Institute of Technology, Karlskrona.

Spierer, D., Rosen, Z., Litman, L. L., \& Fujii, K. (2015). Validation of photoplethysmography as a method to detect heart rate during rest and exercise. Journal of Medical Engineering \& Technology, 39(5), 264-271. https://doi.org/10.3109/03091902.2015.1047536

Terbizan, D. J., Dolezal, B. A., \& Albano, C. (2002). Validity of seven commercially available heart rate monitors. Measurement in Physical Education and Exercise Science, 6(4), 243-247. https://doi.org/10.1207/S15327841MPEE0604 3

Vanderlei, L., Silva, R., Pastre, C., Azevedo, F., \& Godoy, M. (2008). Comparison of the Polar S810i monitor and the ECG for the analysis of heart rate variability in the time and frequency domains. Brazilian Journal of Medical and Biological Research, 41(10), 854-859. https://doi.org/10.1590/S0100-879X2008005000039

Wallen, M., Gomersall, S. R., Keating, S. E., Wisløff, U., \& Coombes, J. S. (2016). Accuracy of heart rate watches: Implications for weight management. PLoS One, 11(5). https://doi.org/10.1371/journal.pone.0154420

Wang, R., Blackburn, G., Desai, M., Phelan, D., Gillinov, L., Houghtaling, P., et al. (2016). Accuracy of wrist-worn heart rate monitors. JAMA Cardiology. 


\section{(c) $(7)(\mathrm{F}$}

This title is licensed under a Creative Commons Attribution-NonCommercial-NoDerivs 3.0 Unported License. 